\title{
EXPERIMENTAL STUDIES OF ARC MOTION BETWEEN TWO PARALLEL RUNNERS WITH SPLITTER PLATES
}

\author{
J. Lu ${ }^{a, *}$, J-J Gonzalez ${ }^{a}$, P. Freton ${ }^{a}$, M. Benmouffok ${ }^{a}$, P. Fort ${ }^{a}$, \\ P. Joyeux ${ }^{b}$, G. DÉPlaude ${ }^{b}$ \\ ${ }^{a}$ Laplace, UMR 5213 CNRS-UPS-INP, Université Paul Sabatier 118 rte de Narbonne, bat3R3, 31062 Toulouse \\ Cedex, France \\ ${ }^{b}$ Hager Electro SAS, 132 Bd d'Europe, 67215 Obernai, France \\ * lu@laplace.univ-tlse.fr
}

\begin{abstract}
In this paper, we present an experimental study in a simplified arc chamber geometry of Low-Voltage Circuit Breaker (LVCB). The influence of vent aperture on arc motion and the influence of splitter plates on arc voltage drop and arc motion are studied. The arc chamber is composed by two parallel arc runners and following the configuration chosen by one or two splitter plates. The experimental setup is completed by a generator (capacitor bench with triggered switch), a high-speed camera and electrical measurements. The arc ignition, arc displacement, arc splitting and arc re-strike have been observed. The results will be described and discussed for different experimental configurations.
\end{abstract}

Keywords: LVCB, splitter plate, arc motion, arc voltage.

\section{Introduction}

The LVCB is an electrical device widely used to protect the power system when a fault current appears in the circuit. The miniature circuit breaker (MCB) is one of LVCB which provides protection of circuit below $1 \mathrm{kV}$ in AC. The electric arc appears during the contacts opening of MCB and should be extinguished rapidly. The process includes the displacement of arc between the arc runners to the quenching chamber which is composed by a series of splitter plates. The technology of arc interruption consists in increase of the arc voltage beyond the network voltage to achieve the current interruption. It is well known that the arc voltage increases by arc elongation. But in the case of MCB, another parameter appears. Due to the voltage drops of the near-electrode regions of splitter plates, the voltage increases too $[1,2]$. This phenomenon is mainly responsible for the current interruption. Therefore, the arc motion (due to elongation) and arc voltage (due to voltage drop) are the most important parameters. The vent aperture plays an important role on the arc motion and splitter plates lead to increase the voltage drop. The study from Shin et al. [3] is devoted to the influence of vent aperture size and repartition on arc motion by arc imaging acquisitions. It is shown that the arc moves further and more quickly in case of larger area of vent opening in the quenching chamber. Nevertheless, the configuration with more vents helps to increase the arc motion velocity. In previous work, McBride et al. [4] studied arc roots motion from moving contact to arc runners. They developed a flexible test apparatus with a fast imaging system to investigate the influence of arc chamber venting on arc motion. The results show that the life time of the arc decreases when the vent area is increased. The arc voltage drop has been studied by experimental and theoretical approaches. Lindmayer et al. [5] and Mutzke et al. [1, 6] proposed to consider an additional voltage drop through the resistivity in the layer of electrodes to well describe the arc splitting process. The solution is governed by U-J relation obtained by experiments. In this relation $\mathrm{U}$ and $\mathrm{J}$ represent respectively the voltage and the local value of the current density in the neighboring cell of the wall (runners and splitter plates). This method allows presenting a realistic arc root voltage description for simulation. Several experiments with one splitter plate in the arc chamber have been analyzed. They found that about $28-30 \mathrm{~V}$ are added to the total voltage after arc splitting process $[1,5,6]$. Further, Yang et al. [7, 8] studied arcing phenomenon by considering a non-linear permeability of ferromagnetic materials. They focus their studies on the influence of eddy currents and the influence of iron metal vapors coming from splitter plates erosion. The experiment has been carried out and the results compared with the simulation. They show that before arc splitting, the anode arc root moves faster than the cathode one. Once the arc reaches the splitter plates, the cathode root moves more quickly than the anode root. In this paper, we present first the experimental setup and the configurations investigated. Then two parameters are experimentally studied: vent configuration on arc motion and the influence of the number of splitter plates on arc voltage evolution. The results are presented and discussed before the conclusion. 


\section{Experimental setup}

\subsection{Configurations}

A simplified arc chamber configuration with two parallel arc runners is used and presented in Figure 1. The chamber allows the presence of zero, one or two splitter plates located in the upstream area. The arc runners and the splitter plates are made by steel covered by copper. A copper wire with a diameter of $0.1 \mathrm{~mm}$ is placed between the two arc runners for arc ignition. Two types of vent condition are used (Figure 2). The copper wire is located $10 \mathrm{~mm}$ from the downstream vent. The distance between the initial position of the copper wire and the splitter plates entry is $30 \mathrm{~mm}$. The position of the copper wire corresponds to the birth place of the conducting channel. The arc can move along $50 \mathrm{~mm}$ from its initial position before reaching the upstream vent which could be totally or partially open ( $15 \%$ of the whole surface) (table 1). Without upstream vent, the arc could reach 20 $\mathrm{mm}$ more before leaving the chamber. The distance between the two main arc runners is $20 \mathrm{~mm}$. The thickness of a splitter plate is $1 \mathrm{~mm}$.

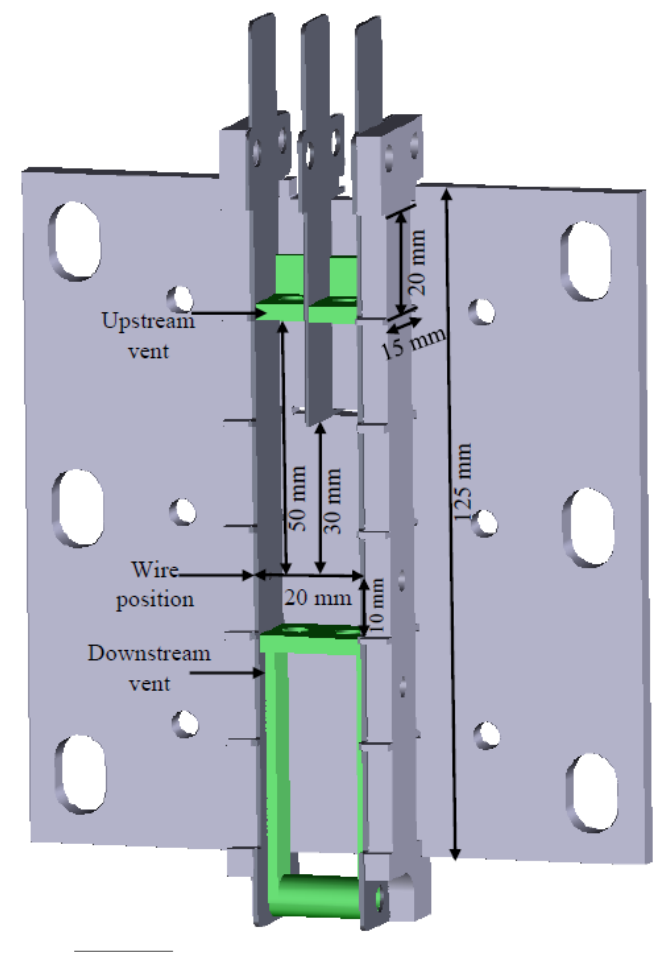

Figure 1. Arc chamber geometry in 3D

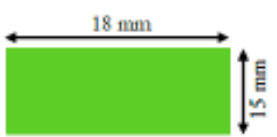

Vent fully closed

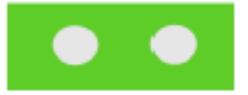

Vent partially open (15\%)
Figure 2. Vent conditions

Figure 3 presents a general view of the experimental setup. It includes a generator composed by capacitors and inductances that can produce a current half-wave of frequency $50 \mathrm{~Hz}$, which has a prospective peak value up to $13 \mathrm{kA}$ with a maximum voltage charge of $450 \mathrm{~V}$ in DC. A high-speed camera (Photron SA5) with a maximal recording speed of 12000 images/s is used with light filters to limit the images saturation. The images obtained by high-speed camera allow observing the arc motion and the splitting process in the arc chamber. The observation requires the use of transparent walls in the experimental device. A differential voltage probe and a Rogowski coil for the electrical measurements are also used. The whole system (Half-wave of the current, high speed camera and electrical measurements) is synchronized by an external Trigger.

\subsection{Test configurations}

Ten configurations have been investigated. The two parameters studied are the number of splitter plates from zero (0 Sp) to two ( $2 \mathrm{Sp})$, and the vent configuration open $(\mathrm{O})$, close $(\mathrm{C})$ or partially open $(\mathrm{PO})$ for the upstream or downstream vent aperture. These cases are presented in Table 1. Each case is reproduced three times. The cases 1 and 2 are devoted to the influence of the number of splitter plates in the configurations $\mathrm{O}-\mathrm{O}$ and $\mathrm{C}-\mathrm{O}$. The configurations $0 \mathrm{Sp}$ and $1 \mathrm{Sp}$ are related to the study of the influence of the vent aperture size. Due to the fact that the arc behavior observed case 3 and case 4 is similar in the configurations $1 \mathrm{Sp}$ and $2 \mathrm{Sp}$, these last results are not presented.

\section{Results and discussion}

Two kinds of measurement are performed with the ten configurations: electrical measurements (current, voltage) and images by high speed camera. The results are difficult to be analyzed and it's better to associate the pictures from the camera with the electrical measurements for the interpretation. So a specific tool was developed with @Matlab software to synchronize acquisitions. The arc roots positions are determined by the method of McBride [9]. Depending on the arc emissivity the tool allows determining the arc roots positions and the mean arc column positions. Sometime the high-speed camera frequency is different with the electrical measurements and the tool allows interpolating to present all the results for the same time.

\subsection{Influence of vent conditions on arc motion}

$\square$ Cases without splitter plate (0 Sp):

Figure 4 and Figure 5 present respectively the average anode root and cathode root positions versus time for the cases without splitter plate. The cases $1,2,3$ allow showing the influence of the downstream vent surface. For those cases the upstream vent is open. The arc motion is only due to loop effects. The arc moves to the upstream position 


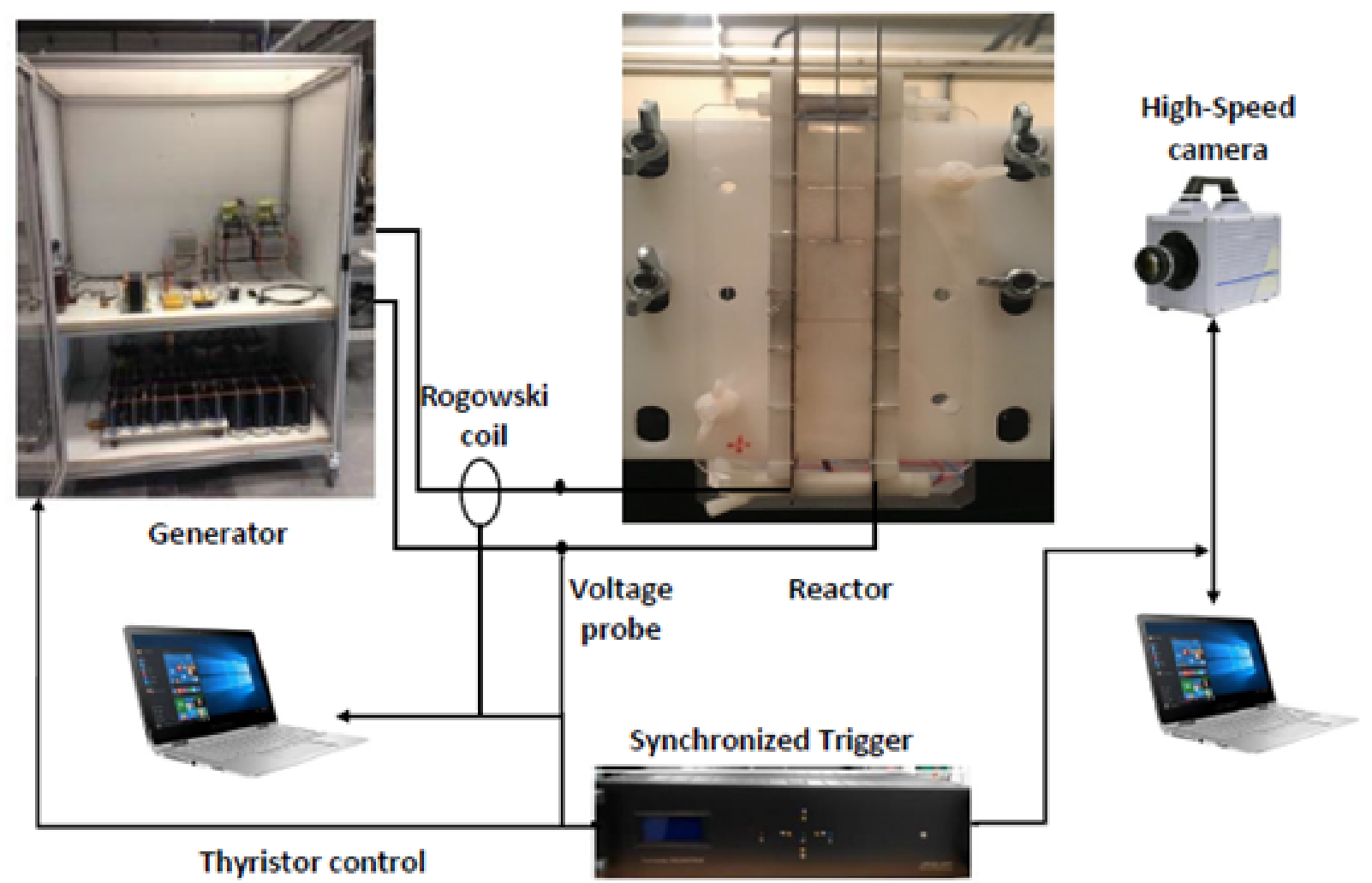

Figure 3. Configuration of experimental setup

\begin{tabular}{lllll}
\hline \hline & Case 1 & Case 2 & Case 3 & Case 4 \\
\hline $0 \mathrm{Sp}$ & O-O & C-O & PO-O & C-PO \\
$1 \mathrm{Sp}$ & O-O & C-O & PO-O & C-PO \\
$2 \mathrm{Sp}$ & O-O & C-O & - & - \\
\hline \hline
\end{tabular}

Table 1. Test configurations

under the influence of the Lorentz forces. Closing progressively the downstream vent leads to an increase of the arc motion. This difference is probably due to pressure effects. Decreasing the vent surface to $85 \%$ (case 3 ) allows increasing the mean velocity from $v_{1}=17.6 \mathrm{~m} / \mathrm{s}$ to $v_{3}=37.6 \mathrm{~m} / \mathrm{s}$.

These velocities are calculated from the mean positions given in Figure 4 and Figure 5. When the downstream vent is totally closed the mean arc velocity is $v_{2}=38.4 \mathrm{~m} / \mathrm{s}$ (case 2 ). In this studied configuration the gas pressure allows increasing by a factor two the mean arc root velocity generated by the Lorentz forces. Of course in this case the plasma viscosity leads to a clogging effect and the pressure acts to push the arc. This phenomenon is possible as the upstream event is open and there is not obstacle to the hydrodynamic flow. On the contrary when the upstream vent is partially closed (case 4) we can observe a decrease of the mean velocity. In those cases the maximum AC current is I = 800A. A comparison between Figure 4 and Figure 5 shows that the behavior is similar for the anodic and cathodic arc roots and that the velocities are in the same order of magnitude.

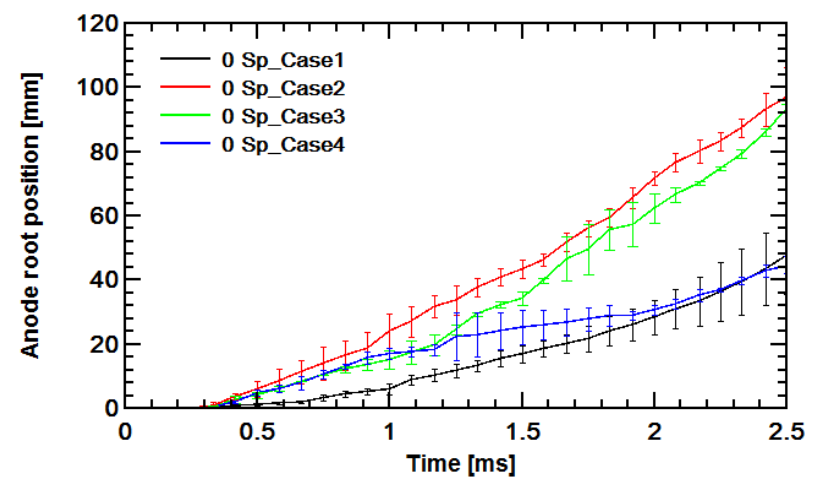

Figure 4. Average anode roots positions for different vent configurations without splitter plate $0 \mathrm{Sp}$

$\square$ Cases with one splitter plate (1 Sp):

The same cases are now considered with the pres- 


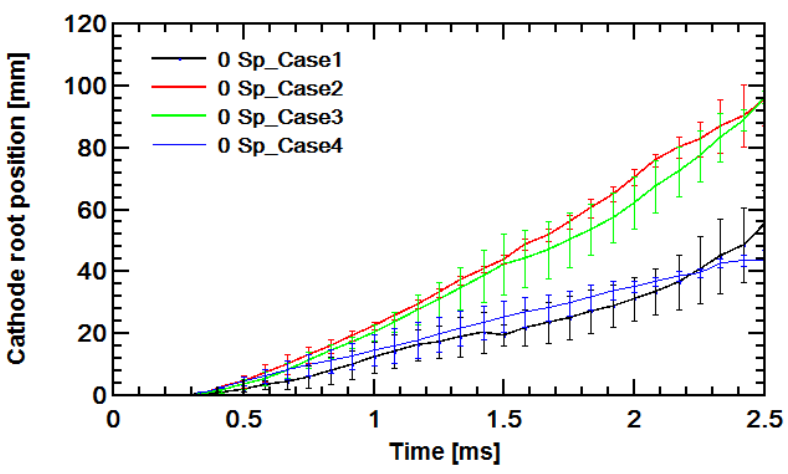

Figure 5. Average cathode roots positions for different vent configurations without splitter plate

ence of one splitter plate in Figure 6 and Figure 7 . The splitter plate is located $30 \mathrm{~mm}$ from the wire position (Figure 1). With the presence of one splitter plate in the arc chamber, the determinations of the arc roots positions are less reproducible and so they present higher error bars. Indeed in case of $1 \mathrm{Sp}$ at the arc commutation beginning multi attachements exist with sometimes a current path in front of the splitter plates. After the commutation two half arcs exist on each splitter plates faces. These two arcs are not located at the same position and additional loop effects due to the current path in the splitter plates appear. All these phenomenon lead to increase the error bars. Similar behavior on arc root velocities observed without splitter plate is found with $1 \mathrm{Sp}$. Figure 6 and Figure 7 represent the mean arc root positions on the runners in the configuration $1 \mathrm{Sp}$. The splitter plates position is represented by an horizontal dashed line.

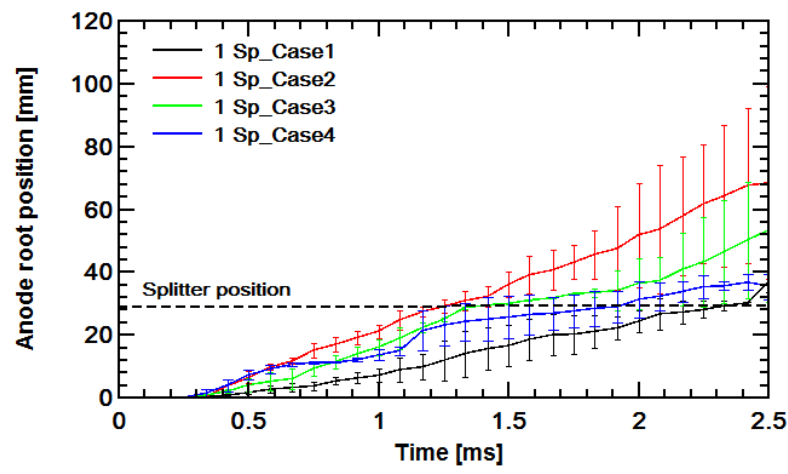

Figure 6. Average anode roots positions for different vent configurations with one splitter plate

Figure 6 shows that the mean anodic arc root velocities are lower when one splitter plate is present in comparison to Figure 4. This behavior is observed for the four cases. We can observe a real difficulty for the arc root at the anode to reach the splitter plate in case $1(\mathrm{O}-\mathrm{O})$. In the same time we observe a slope change for the case 3 (PO-O) indicating a stagnation and a not easy configuration for the arc to overcome the splitter plate. In the two cases where the downstream vent is closed, we do not observe slope change on the curves, indicating that the difficulty for the arc to overcome the splitter is compensated by pressure effects.

When we look to the mean cathodic arc root positions, it seems that the arc roots velocities are the same as in the cases without splitter plate. Exceptfor the case 1 all the cases seem to be affected by the splitter presence and arc stagnations are observed. In conclusion it seems that the splitter presence mainly affects the anodic arc root positions leading to a decrease of the velocities.

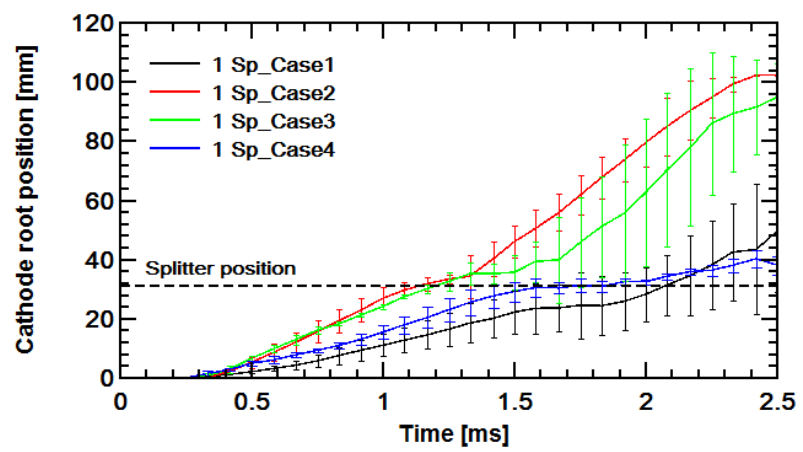

Figure 7. Average cathode roots positions for different vent configurations with one splitter plate

\subsection{Influence of splitter plates on arc voltage}

In this section we study the influence of the splitter plate presence on the electrical measurements (intensity, arc voltage). Figure 8 presents the total voltage measured between the two runners. One peak located at time $\mathrm{t}=0.33 \mathrm{~ms}$ is observed, it corresponds to the copper wire explosion used for the arc ignition. After the wire explosion, in the case without splitter plate, the voltage drop keeps nearly constant with a value around $70 \mathrm{~V}$. This voltage value corresponds to the sum of the arc column voltage and the values of the anodic and cathodic sheath voltages.

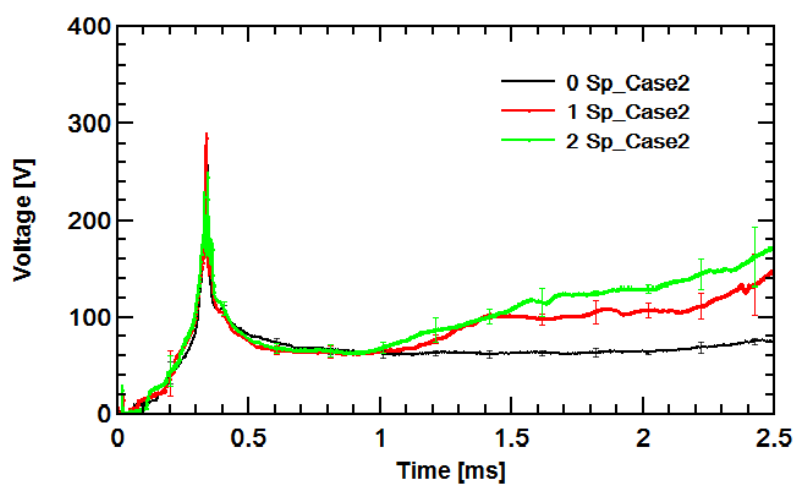

Figure 8. Average voltages for case 2 with zero, one and two splitter plates

In cases of one and two splitters after $1 \mathrm{~ms}$, the voltage increases. We have seen in Figure 6 and Figure 7 that the splitter plate was reached in the time interval 
1 to $1.3 \mathrm{~ms}$. During this lapse of time the voltage increases due to the bent of the arc increasing its length, this is why we do not really observe difference between the configurations with one or two splitters. After 1.3 ms, the two curves present different evolutions with an higher voltage in case of two splitters. The difference between the two curves is around $30 \mathrm{~V}$. Figure 6 and Figure 7 have shown different positions versus time for the anodic and cathodic arc roots. This indicates that the arc length changes with time and that the arc is not perpendicular to the runners. It is so difficult to discuss the additional voltage value in more detail which is a combination of changes in arc length and additional drops due to the splitters presence. In Figure 9 we have represented the current intensity evolution versus time for zero, one and two splitters corresponding to the case $2(\mathrm{C}-\mathrm{O})$. In this same figure the prospective current intensity corresponding to the applied current without limitation is plotted. The current limitation occurs close to $1 \mathrm{~ms}$ and $1.3 \mathrm{~ms}$ as indicated previously. From the curve of the prospective current, we can note a greater limitation following the number of splitter plates.

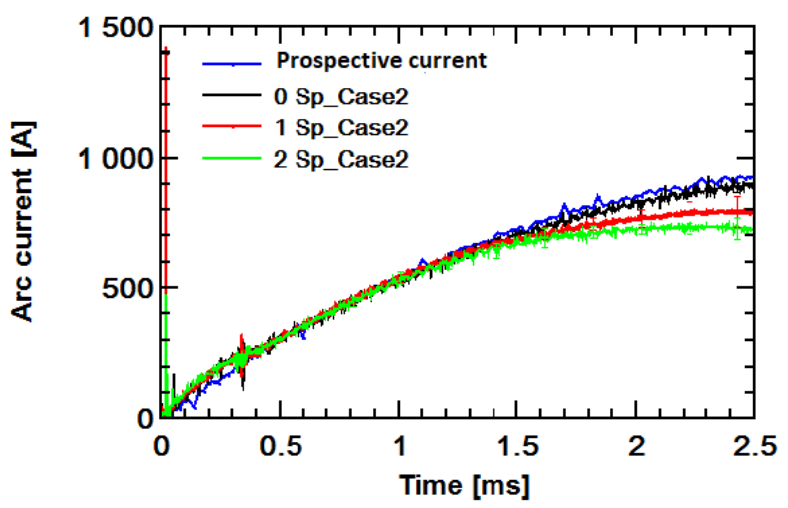

Figure 9. Average current intensity versus time. Case 2 with zero one and two splitter plates.

\section{Conclusion}

In this paper the arc behavior in a simple chamber of LVCB is studied. The experimental setup is presented. The geometrical parameters investigated are related to the area of the vents and to the number of splitter plates. The results show that the arc ignition occurs at around $0.3 \mathrm{~ms}$. Depending on the vent surface the pressure effect is highlighted. Indeed in the openopen $(\mathrm{O}-\mathrm{O})$ configuration the arc moves only under the influence of the Lorentz forces due to loop effects. When the downstream area is closed or partially closed the arc velocity increases. This can be explained by pressure effects. The mean arc velocity is multiplied by a factor two between the configurations O-O and C-O. Depending on the number of splitter plates, the total voltage increases leading to a current limitation. The total voltage is directly correlated to the number of splitter plates. Due to the changes in arc length following the configuration and the fact that the arc velocities are also not the same, the arc positions between two configurations differ. It is so difficult to estimate deeper the splitter contribution, nevertheless a contribution of around $30 \mathrm{~V}$ by splitter is estimated.

\section{References}

[1] A. Mutzke, T. Ruther, M. Lindmayer, and M. Kurrat. Arc behavior in low-voltage arc chambers. European Physical Journal Applied Physics, 49, 2010. doi:10.1051/epjap/2010001.

[2] J. Lu, G. Déplaude, F. Freton, J.-J. Gonzalez, and P. Joyeux. Experimental and simulation studies on the voltage drop of arc in low-voltage circuit breaker. European Physical Journal Applied Physics, 6(3), 2019. doi:10.14311/ppt.2019.3.256.

[3] D. Shin, I. O. Golosnoy, T. G. Bull, and J. W. McBride. Experiment study on the influence of vent aperture size and distrubution on arc motion and interruption in low-voltage switching devices. 4th International Conference on Electric Power Equipment-Switching Technology (ICEPE-ST), 2017. doi:10.1109/ICEPE-ST . 2017.8188830.

[4] J. W. McBride, K. Pechrach, and P. M. Weaver. Arc root commutation from moving contacts in low voltage devices. IEEE Transactions on Components and Packaging Technologies, 24(3), 2001. doi:10.1109/6144.946475.

[5] M. Lindmayer, E. Marzahn, A. Mutzke, T. Ruther, and S. M. The process of arc splitting between metal plates in low voltage arc chutes. IEEE Trans. CPMT, 29(2), 2006. doi:10.1109/HOLM. 2004.1353090.

[6] A. Mutzke, T. Ruther, M. Kurrat, M. Lindmayer, and E. D. Wilkening. Modeling the arc splitting process in low voltage arc chutes. 53 rd IEEE Holm Conf. on Electrical Contacts Pittsburgh, 2007. doi: 10.1109/HOLM. 2007.4318213.

[7] F. Yang, M. Rong, and Y. Wu. Numerical simulation of the eddy current effects on the arc splitting process. Plasma Science and Technology, 14(11), 2012. doi:10.1109/TPS.2012.2050703.

[8] F. Yang, M. Rong, and Y. Wu. Numerical analysis of arc characteristics of splitting process considering ferromagnetic plate in low-voltage arc chamber. IEEE Trans. Plasma Sci., 43(43), 2010. doi : 10.1088/0022-3727/43/43/434011.

[9] J. W. McBride and P. M. Weaver. A review of arcing phenomena in low voltage current limiting circuit breakers. IEE Proc. Sci. Meas. and Tech., 1(23), 2001. doi:10.1049/ip-smt: 20010185. 